\title{
A TRANSMISSION LINE MODEL FOR THE SPHERICAL BELTRAMI PROBLEM
}

\author{
$\underline{\text { C. D. Papageorgiou }}^{1}$, T. E. Raptis ${ }^{2}$
}

\author{
${ }^{1}$ Dept. of Electrical \& Electronic Engineering, \\ National Technical University of Athens, Greece \\ E-mail: chrpapa@central.ntua.gr \\ ${ }^{2}$ Division of Applied Technologies , \\ National Centre for Science and Research "Demokritos", \\ Patriarchou Grigoriou \& Neapoleos, Athens, Greece \\ E-mail: rtheo@dat.demokritos.gr
}

\begin{abstract}
We extend a previously introduced model for finding eigenvalues and eigenfunctions of PDEs with a certain natural symmetry set based on an analysis of an equivalent transmission line circuit. This was previously applied with success in the case of optical fibers [8], [9] as well as in the case of a linear Schroedinger equation [10], [11] and recently in the case of spherical symmetry (Ball Lightning) [12]. We explore the interpretation of eigenvalues as resonances of the corresponding transmission line model. We use the generic Beltrami problem of non-constant eigen-vorticity in spherical coordinates as a test bed and we locate the bound states and the eigen-vorticity functions.
\end{abstract}

\section{Introduction}

The notion of a Beltrami field appears first in hydrodynamics in the studies of Eugenio Beltrami [1] at the end of the $19^{\text {th }}$ century where it has found widespread application in vortical fluid states with a characteristic helical geometry. Its rediscovery in modern electromagnetism took place at the middle of the $20^{\text {th }}$ century when Lundquist [2] proposed the same model for galactic magnetic fields where the field appears to be parallel to its own rotation (neglecting displacement current). Later, Lust and Schluter [3] and Chandrasekhar and Woltjier [4] proposed a theoretical justification of these states in terms of magnetic plasma equilibrium. Several types of solutions are reviewed in [6] and [7].

Nowadays, the Beltrami equation serves as a model of

a) "Force-Free Fields" for which the magnetic part of the Lorentz force disappears ( $\mathbf{J} / / \mathbf{B})$ in equilibrated plasma and

b) for parallel $\mathbf{E} / / \mathbf{B}$ components when applied to the vector potential directly (in which case we get $\mathbf{E}=-\omega \mathbf{A}, \mathbf{B}=\lambda(\mathbf{r}, t) \mathbf{A})$.

The Beltrami problem is defined by at least two and at most three PDEs given by

$$
\begin{aligned}
& \nabla \times \mathbf{A}=\lambda(\mathbf{r}, t) \mathbf{A} \\
& \nabla \lambda \bullet \mathbf{A}=\nabla \bullet \mathbf{A}=0
\end{aligned}
$$

The second condition is usefull in applications in electromagnetism when $\mathbf{A}$ stands either for the vector potential or the magnetic field. Applying twice the rotation operator in the first of (1) gives the most general representation of the non-linear inhomogeneous Helmholtz operator for Beltrami flows as 


$$
\nabla^{2} \mathbf{A}-\lambda^{2}(\mathbf{r}, t) \mathbf{A}=\nabla \lambda \times \mathbf{A}
$$

In the case of constant $\lambda$ the inhomogeneous part is of course zero. Solutions of this class are usually termed linear "Trkalian" fields as they were first studied extensively by Viktor Trkal [5].

In the next paragraph we turn to equation (1) and attempt to locate eigen-modes in terms of the orthogonal basis of vector spherical harmonics. In section 2, we perform the expansion and derive the associated ODEs and in section 3, we introduce the transmission line model for this system. In section 4 , we solve the model for the associated angular momentum eigenvalue and we approximate the specific eigen-mode for a "bound state".

\section{Spherical expansion of the Beltrami equation}

In what follows we restrict attention to purely radial functions for the eigen-vorticity $\lambda(\mathrm{r})$. We introduce the usual basis of Vector Spherical Harmonics as $\left\{\overrightarrow{\mathrm{Y}}=\mathbf{r} \Psi_{l m}, \vec{\Psi}=r \nabla \Psi_{l m}, \vec{\Phi}=\mathbf{r} \times \nabla \Psi_{l m}\right\}$ and we seek an appropriate decomposition of the field $\mathbf{A}$ and its eigen-vorticity $\lambda$. Then there must be a unique reference frame for which both equations ( $1 \mathrm{a}-\mathrm{b})$ can be satisfied by taking

$$
\mathbf{A}=\sum_{l=0}^{\infty} \sum_{m=-l}^{l}\left[a_{l m}(r) \overrightarrow{\mathrm{Y}}_{l m}+b_{l m}(r) \vec{\Psi}_{l m}+c_{l m}(r) \vec{\Phi}_{l m}\right]
$$

Then condition $\nabla \mathbf{A}=0$ is satisfied if

$$
\begin{aligned}
& \nabla \mathbf{A}=\sum_{l=0}^{\infty} \sum_{m=-l}^{l}\left[\left(\dot{a}_{l m}+\frac{2 a_{l m}}{r}\right)-\frac{l(l+1)}{r} b_{l m}(r)\right] \Psi_{l m}=0 \\
& \dot{a}_{l m}+\frac{2 a_{l m}}{r}-\frac{l(l+1)}{r} b_{l m}=0
\end{aligned}
$$

while the choice of the $\lambda$ function is restricted by $\nabla \lambda \bullet \mathbf{A}=0$. For a purely radial $\lambda$ function it suffices to take the additional condition that the radial part of $\boldsymbol{A}$ is set to zero which is a very stringent condition on the coefficients of (5). We note however that in the more general case we may allow for violations of condition (1b). In electromagnetic applications we may instead assume the presence of static charges satisfying the Lorenz gauge $\partial_{t} \phi=-c \nabla \mathbf{A}=c \lambda^{-1} \nabla \lambda \bullet \mathbf{A}$.

From now on we will deal solely with the first condition (1a) for the rotation of $\mathbf{A}$. We now turn back to the first of (1) for the rotation operator which obtains

$$
\nabla \times \mathbf{A}=\sum_{l=0}^{\infty} \sum_{m=-l}^{l}\left\{-\frac{l(l+1)}{r} c_{l m} \overrightarrow{\mathrm{Y}}_{l m}-\left(\dot{c}_{l m}+\frac{c_{l m}}{r}\right) \vec{\Psi}_{l m}+\left(\dot{b}_{l m}+\frac{b_{l m}}{r}-\frac{a_{l m}}{r}\right) \vec{\Phi}_{l m}\right\}
$$

For the rotation to be parallel to $\lambda \boldsymbol{A}$ we should have

$$
\begin{aligned}
& -\frac{l(l+1)}{r} c_{l m}=\lambda a_{l m} \\
& \dot{c}_{l m}+\frac{c_{l m}}{r}+\lambda b_{l m}=0 \\
& \dot{b}_{l m}+\frac{b_{l m}}{r}-\frac{a_{l m}}{r}-\lambda c_{l m}=0
\end{aligned}
$$


Using the first of (9) results in the ODE system

$$
\begin{aligned}
& (r b)^{\prime}=\left[\lambda-\frac{l(l+1)}{r^{2} \lambda}\right](r c) \\
& (r c)^{\prime}=-\lambda(r b)
\end{aligned}
$$

\section{The Transmission Line model}

At this section we introduce an isomorphism between the system (10) and a lossless transmission line via the mapping

$$
\begin{aligned}
& V=r c \rightarrow \frac{d V}{d r}=-\mathbf{j} \lambda I \\
& \mathbf{j} I=r b \rightarrow \frac{d I}{d r}=-\frac{\gamma^{2}}{\mathbf{j} \lambda} V
\end{aligned}
$$

where we have introduced the propagation constant $\gamma^{2}=\frac{L^{2}}{r^{2}}-\lambda^{2}, L=l(l+1)$. Equivalently we may define the transmittances to be

$$
\begin{aligned}
& \mathrm{Z}_{1}=-\mathbf{j} \lambda \\
& \mathrm{Y}_{1}=\mathbf{j} \frac{\gamma^{2}}{\lambda}
\end{aligned}
$$

Then the complex impedance is defined through

$$
\begin{aligned}
& \bar{Z}=\sqrt{\frac{Z_{1}}{Y_{1}}}=\mathbf{j} \frac{\lambda}{\gamma}=\mathbf{j} \sqrt{\frac{L^{2}}{r^{2} \gamma^{2}}-1} \\
& Z_{1} Y_{1}=\gamma^{2}
\end{aligned}
$$

From standard transmission line theory we have that propagation is defined for

$$
\gamma^{2}<0 \quad \text { or } \quad r>\frac{|\lambda|}{\sqrt{l(l+1)}}
$$

while damping (evanescent modes) for

$$
\gamma^{2}>0 \text { or } r<\frac{|\lambda|}{\sqrt{l(l+1)}}
$$

An element of such a transmission line corresponds to a lumped-element model of the initial domain $[0, \infty]$ of the radius $r$ into a set of mesh points $r_{0}, r_{1}, \ldots, r_{n}$ with each element interpolating into the arbitrary intervals $\left[r_{n}, r_{n+1}\right]$.We then take each element to correspond to a segment of infinitesimal width $\Delta r=r_{n+1}-r_{n}$ for which the $\gamma$ factor can be taken approximately constant. For such an element we may take the equivalent relations 


$$
\left[\begin{array}{c}
V\left(r_{n}\right) \\
I\left(r_{n}\right)
\end{array}\right]=\left[\begin{array}{ll}
\cosh \left(\xi_{n}\right) & \sinh \left(\xi_{n}\right) \\
\sinh \left(\xi_{n}\right) & \cosh \left(\xi_{n}\right)
\end{array}\right]\left[\begin{array}{c}
V\left(r_{n+1}\right) \\
I\left(r_{n+1}\right)
\end{array}\right]
$$

where we have put $\xi_{n}=\gamma(n) \Delta r$. The value of the impedance at each point of the line will be given by the recursive formula

$$
Z\left(r_{n}\right)=\frac{V\left(r_{n}\right)}{I\left(r_{n}\right)}=\bar{Z}_{n} \frac{Z\left(r_{n+1}\right)+\bar{Z}_{n} \tanh \left(\xi_{n}\right)}{Z\left(r_{n+1}\right) \tanh \left(\xi_{n}\right)+\bar{Z}_{n}}
$$

and the boundary conditions are defined through the respective limits $Z(r \rightarrow 0), Z(r \rightarrow \infty)$ based on the assumption that the asymptotic limit of a lossless transmission line corresponds to the fixed point of (19) given by $\bar{Z}_{n}$ according to (16).

\section{Trapped modes and bound states}

The form of the propagation constant leads to the possibility of "trapped" waves and their respective eigenmodes. A necessary condition for trapping is to have at least one region $r_{1} \leq r \leq r_{2}$ where $\gamma^{2}(r)<0$ allowing propagation and two disparate regions $r<r_{1}, r>r_{2}$ with $\gamma^{2}(r)>0$ where only evanescent modes exist.

This situation is reminiscent of the bound states of quantum mechanical systems with the function $\lambda^{2}(r)$ playing a role analogous to that of the potential in the radial Schroedinger equation. Evanescent modes are then analogous to the scattered wave functions above a threshold. On the other hand, a constant value of $\lambda$ allows only a simple root $r_{3}=\sqrt{l(l+1)} / \lambda_{0}$ beyond which propagation is allowed up to the infinity. We will next evaluate one such example of an eigenvorticity function supporting a trapped mode.

According to a standard technique introduced in [11] and [12], a method for locating the eigenvalues and compute the associated eigen-mode is based on a simple resonance theory for transmission lines. In this viewpoint, we may take the bound state to correspond to an area where ordinary resonance takes place through the condition $Z_{L}=Z_{C}$ between the "capacitive" and "inductive" elements in the imaginary part of the respective complex impedance. This can effectively be restated as

$$
\operatorname{Im}\left\{Z^{+}\left(r_{c}\right)+Z^{-}\left(r_{c}\right)\right\}=0
$$

Evolution of the above quantity in the region $\left[r_{1}, r_{2}\right]$ prescribes also the associated eigen-mode. In order to locate a certain mode for a given value of angular momentum we iterate the mapping (21) forward and backward $\left(r_{n} \rightarrow-r_{n}\right)$ in the interval $\left[r_{1}, r_{2}\right]$ and expand the interval until we find a root of (22).

A simple choice of a $\gamma$ function with a trapping region is given by

$$
\gamma^{2}=\frac{L^{2}\left(r-r_{1}\right)\left(r-r_{2}\right)}{r^{4}+r_{1} r_{2} r}
$$




$$
\begin{aligned}
& \lambda^{2}=\frac{L^{2}}{r\left(R_{1} r^{2}+R_{2}\right)}, \\
& R_{1}=1 /\left(r_{1}+r_{2}\right), R_{2}=r_{1} r_{2} / R_{1}
\end{aligned}
$$

In Fig. 1 we show the exact form of the propagation factor while for the eigen-vorticity we show that $\lambda^{2}$ is positive definite everywhere. In Fig. 2 and 3 we show the evolution of the difference of the imaginary parts for $l=1$ and $l=2$ and boundary conditions $Z(0)=0, Z(\infty) \cong \mathbf{j} 10^{9}$.

\section{Beltrami solutions through the Lorentz group}

From the previous analysis a fundamental observation occurs based on the hyperbolic relation connecting the triplet $\{\gamma, \lambda, L / r\}$ which can be written as

$$
\gamma^{2}(r)+\lambda^{2}(r)-\frac{L^{2}}{r^{2}}=0
$$

Going to the transformed variables

$$
\begin{aligned}
& X=\gamma^{2}(r) \\
& Y=\lambda^{2}(r) \\
& T=\mathbf{j} / r
\end{aligned}
$$

leads to the Lorentzian manifold $\mathrm{M}(2,1)$ with the usual Minkowski metric of signature $(1,1,-1)$ that has an invariant "null" length (virtual photon path)

$$
s^{2}=X^{2}+Y^{2}-(L T)^{2}=0
$$

and a "speed-of-light" $\mathrm{c}=L$.

This means that given a set of "core" functions $\left\{\gamma_{0}, \lambda_{0}\right\}$ and a angular momentum value $L$ we can produce an infinite family of deformed solutions which are connected by both proper and improper Lorentz transforms. Thus, for the choice represented by (23) we may find a continuous trajectory passing through different families of functions $\left\{\gamma_{n}, \lambda_{n}\right\}$ by a direct application of the generic linear transformation $(X, T) \stackrel{\Lambda(L)}{\longrightarrow}\left(X^{\prime}, T^{\prime}\right):\left(\gamma_{v}, \lambda_{v}\right) \stackrel{\Lambda(L)}{\longrightarrow}\left(\gamma_{v+1}, \lambda_{v+1}\right)$ where the matrix $\Lambda(L)$ is parametrized according to the four conjugacy classes of the full Lorentz group (elliptic, hyperbolic, parabolic and Loxodromic) thus leading to different families of solutions.

\section{Conclusions}

We showed that a general class of solutions of the spherical Beltrami problem can be found in terms of vector spherical harmonics of which the resulting system is equivalent with a generic lossless transmission line. When applied to a specific choice of eigen-vorticity function it allows finding the set of parameters necessary for each mode to exist. We have given explicitly an example of such a function for which trapped modes can exist in a region. We believe that this phenomenon deserves further examination especially with recourse to electromagnetic applications.

\section{References}


[1] E. Beltrami, Rend. Inst. Lombardo Acad. Sci. Lett. 22 (1889) 121-131

[2] S. Lundquist, Ark. Fys. 2 (1951) 361

[3] R. Lust, A. Schluter, Z. Astrophys. 34 (1954) 263

[4] S. Chandrasekhar, P. C. Kendall, Astrophys. J. 126 (1957) 126

[5] A. Lakhtakia, Czechoslovak J. Phys. 44(2) (1994) 89 - 96

[6] H. Zaghloul, O. Barajas, Am. J. Phys. 58(8) (1990) 783-788

[7] G. E. Marsh, "Force-Free Magnetic Fields", World Sci. (1996)

[8] C.D. Papageorgiou, JD Kanellopoulos, J. Phys. A: Math. Gen. 15 (1982)

2569-2580.

[9] C. D. Papageorgiou, A. D. Raptis, Comp. Phys. Com. 43 (1987) 325 - 328

[10] C. D. Papageorgiou et al., J. Comp. App. Math. 29 (1990) 61-67

[11] C. D. Papageorgiou et al.,J. Comp. Phys. 88(2) (1990) 477-483.

[12] C. D. Papageorgiou, T. E. Raptis, under preparation.

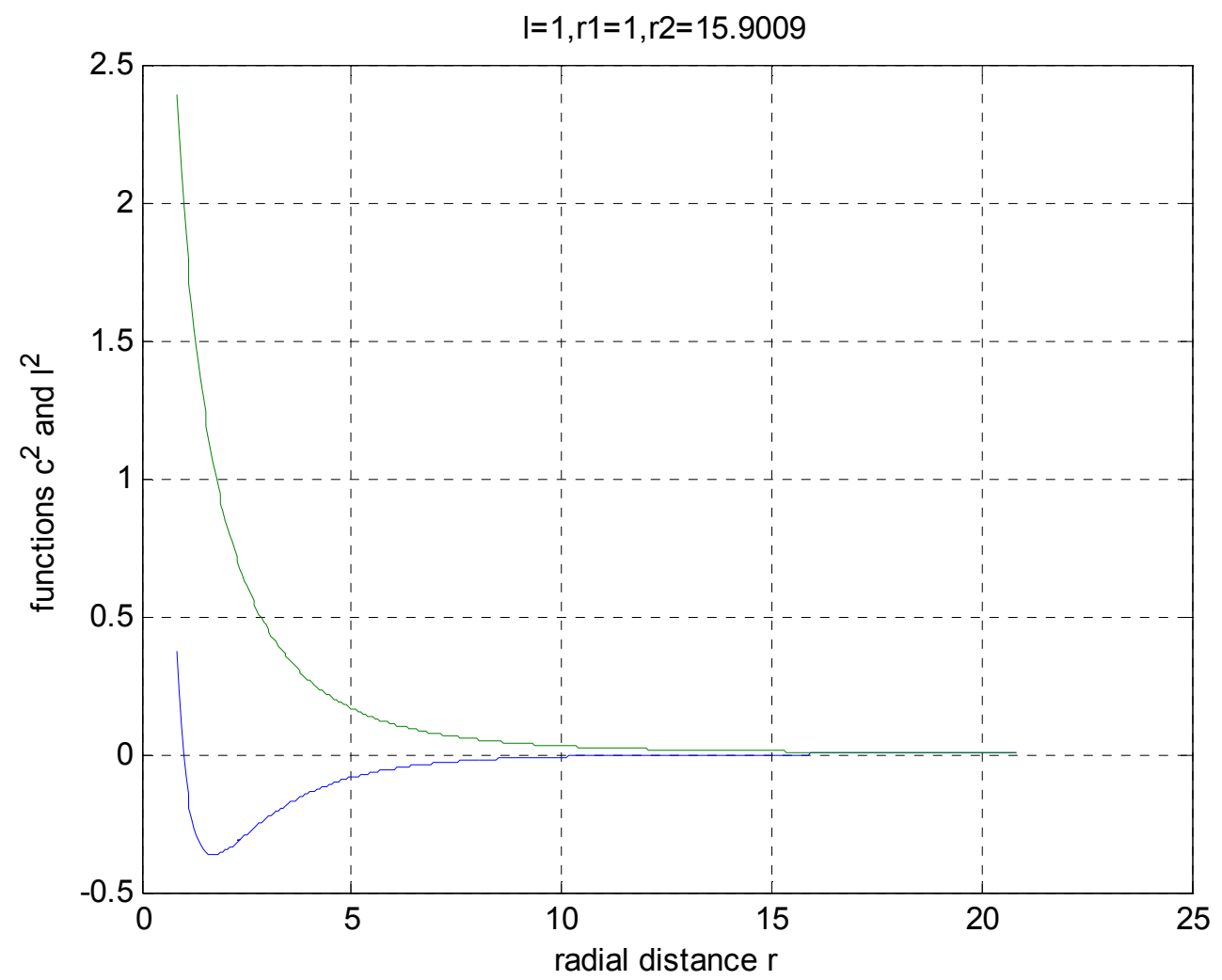

Fig. 1 


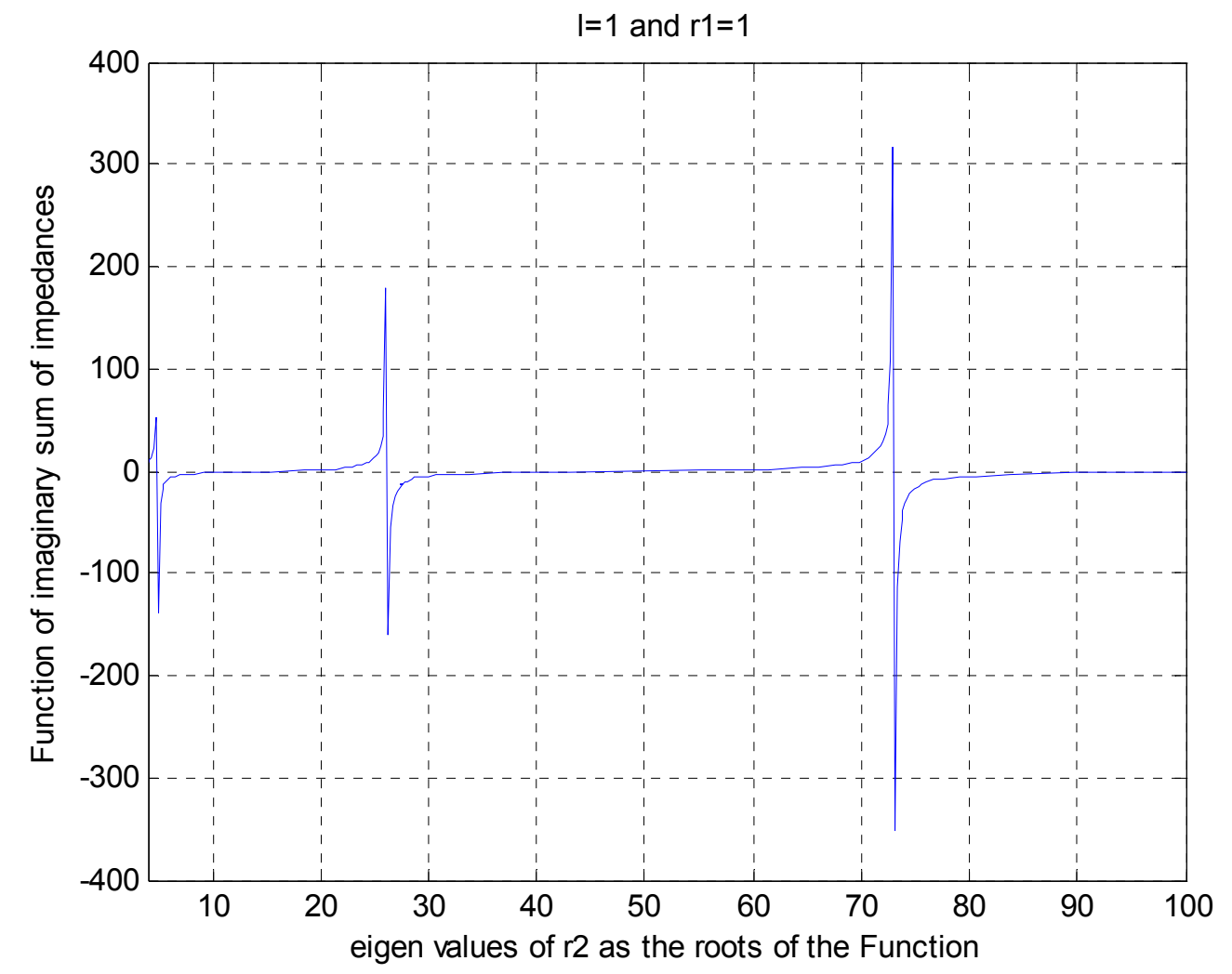

Fig. 2 


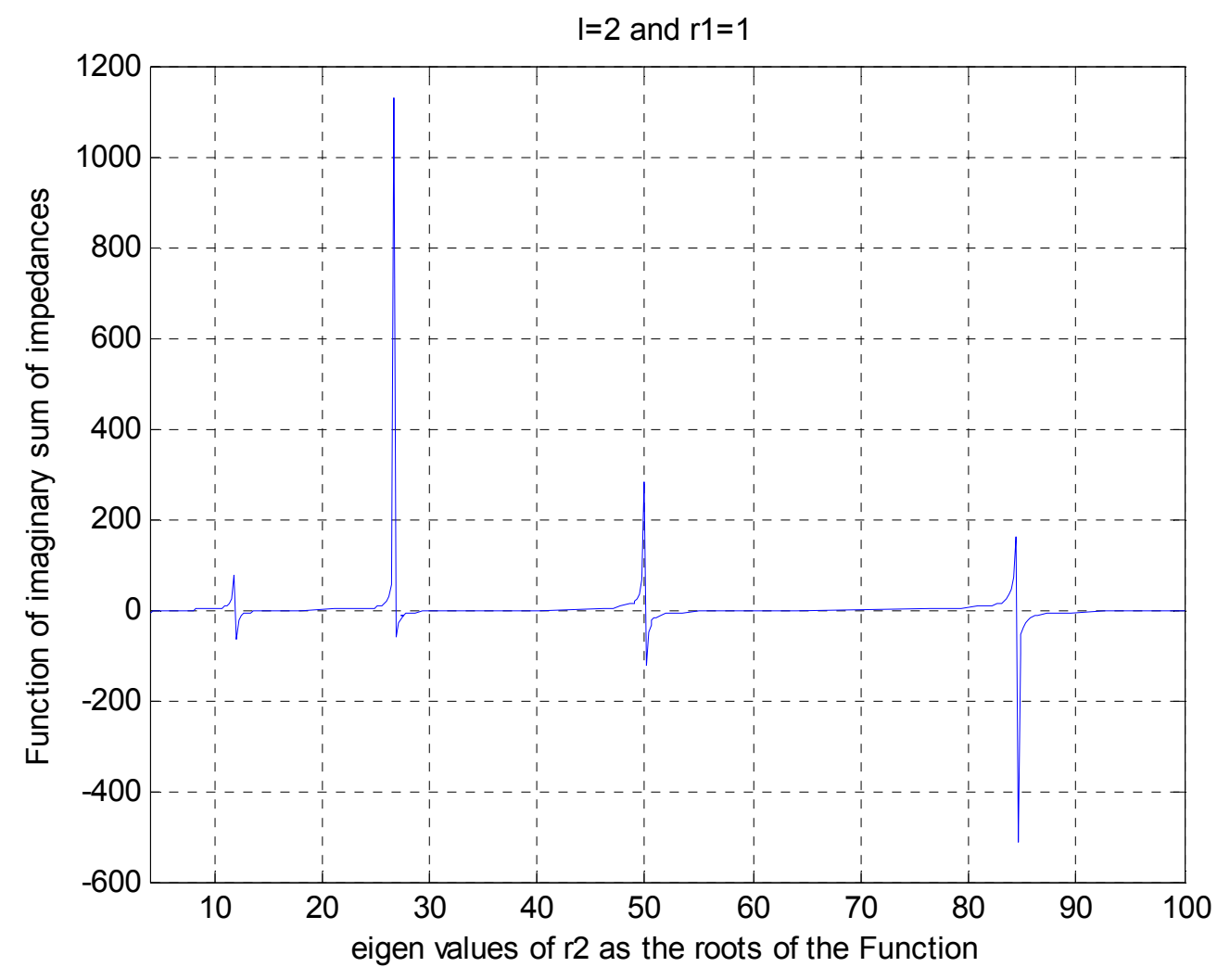

Fig. 3 